\section{Plastia desmembrada de la unión ureteropiélica laparoscópica asistida por robot}

González-Serrano A, Cortez-Betancourt R, Alías-Melgar A, Carreño-De la Rosa F, Botello-Gómez PJ, Trujillo-Vázquez El, Ordóñez-Campos E, RodríguezAlvarado RC

\section{Resumen}

ANTECEDENTES: en $63 \%$ de los pacientes con estenosis de la unión ureteropiélica existe un vaso polar inferior, que siempre se ha considerado "aberrante", y como cruza por delante del uréter reduce su luz. Estos vasos representan variantes anatómicas de vasos provenientes de la aorta abdominal y la misma arteria renal.

CASO CLÍNICO: paciente femenina de 26 años de edad, con antecedentes de hipertensión arterial de un año de evolución, dolor en la fosa renal derecha e infección urinaria recurrente. En la urotomografía se evidenció, siempre en el lado derecho: hidronefrosis y vaso aberrante en torno a la unión ureteropiélica, curva obstructiva en la unidad renal, postgamagrama renal con diurético. Con el sistema quirúrgico Da Vinci ${ }^{\circledR}$ se realizó una plastia ureteropiélica desmembrada, sin complicaciones. El tiempo quirúrgico fue de 110 minutos. La paciente fue dada de alta del hospital al segundo día. El catéter JJ se retiró a las seis semanas y se realizó nuevo gamagrama un mes después del retiro del catéter.

CONCLUSIÓN: la pieloplastia asistida por robot es un método mínimamente invasivo, seguro y efectivo para corregir la estenosis ureteropiélica.

PALABRAS CLAVE: estenosis, unión ureteropiélica, robot, da Vinci.

Rev Mex Urol. 2017 January;77(1):79-84.

\section{Robotic-assisted laparoscopic dismembered ureteropyeloplasty.}

González-Serrano A, Cortez-Betancourt R, Alías-Melgar A, Carreño-De la Rosa F, Botello-Gómez PJ, Trujillo-Vázquez El, Ordóñez-Campos E, RodríguezAlvarado RC

\section{Abstract}

BACKGROUND: In $63 \%$ of the patients with ureteropelvic junction obstruction, there is a so-called "aberrant" vessel that crosses in front of the ureter, reducing its lumen. These vessels are anatomic
Departamento de Urología, Centro Médico Naciona 20 de Noviembre, Instituto de Seguridad y Servicios Sociales de los Trabajadores del Estado, Ciudad de México, México.

Recibido: agosto 2016

Aceptado: enero 2017

Correspondencia

Adolfo González Serrano

agonzalezuro@gmail.com

Este artículo debe citarse como

González-Serrano A, Cortez-Betancourt R, AlíasMelgar A, Carreño-De la Rosa F, Botello-Gómez PJ, Trujillo-Vázquez El, Ordóñez-Campos E, RodríguezAlvarado RC. Plastia desmembrada de la unión ureteropiélica laparoscópica asistida por robot. Rev Mex Urol. 2017 ene;77(1):79-84. 
variants of vessels arising from the abdominal aorta and the renal artery.

CLINICAL CASE: A 26-year-old woman had a past history of high blood pressure of one-year progression, pain in the right renal fossa, and recurrent urinary infections. A computed tomography urogram identified right hydronephrosis and an aberrant vessel at the level of the ureteropelvic junction on the right side. A kidney scintigram with a diuretic revealed an obstructive curve in the right kidney unit. Robotic-assisted dismembered ureteropyeloplasty was performed with no complications, using the da Vinci ${ }^{\circledR}$ Surgical System. Surgery duration was $110 \mathrm{~min}$. The patient was released from the hospital on the second postoperative day. The double-J catheter was removed after six weeks and a repeat scintigram was carried out one month after catheter removal.

CONCLUSION: Robotic-assisted ureteropyeloplasty is a safe and effective minimally invasive method for correcting ureteropelvic junction obstruction.

KEY WORDS: Stenosis; Ureteropelvic junction; Robot; da Vinci
Departamento de Urología, Centro Médico Nacional 20 de Noviembre, Instituto de Seguridad y Servicios Sociales de los Trabajadores del Estado, Ciudad de México, México.

Correspondence Adolfo González Serrano agonzalezuro@gmail.com

\section{ANTECEDENTES}

En $63 \%$ de los pacientes con estenosis de la unión ureteropiélica existe un vaso polar inferior, que siempre se ha considerado "aberrante", y como cruza por delante del uréter reduce su luz. Estos vasos representan variantes anatómicas de vasos provenientes de la aorta abdominal y la misma arteria renal. ${ }^{1}$

La simple existencia de la estenosis ureteropiélica no implica tratamiento. Se saben las indicaciones para su corrección con la cirugía abierta y son semejantes a la cirugía robótica. Las indicaciones son: hidronefrosis progresiva, deterioro progresivo de la función renal, infecciones urinarias recurrentes en el contexto de la obstrucción y síntomas como el dolor, náusea, vómito y hematuria. De igual forma existen contraindicaciones absolutas y relativas para el procedimiento. Las primeras se resumen a la infección urinaria no tratada, las segundas abarcan la cirugía abdominal previa, una pelvis intrarrenal pequeña, un segmento estenótico largo y pacientes menores de $6 \mathrm{~kg}{ }^{2}$

En nuestro país la cirugía robótica representa una alternativa terapéutica de reciente inicio, en constante desarrollo y es una realidad para algunos centros hospitalarios en la actualidad.

En nuestro caso, el CMN 20 de Noviembre ha iniciado desde el año 2015 a realizar cirugías asistidas por el sistema quirúrgico da Vinci ${ }^{\circledR}$ obteniendo buenos resultados.

El objetivo de este trabajo es mostrar una variante de la plastia ureteropiélica desmembrada laparoscópica asistida por robot y los resultados obtenidos con este procedimiento. 


\section{CASO CLÍNICO}

Se trata de un paciente femenino de 26 años con historia de hipertensión arterial de 1 año de evolución en tratamiento con nifedipino $20 \mathrm{mg}$ c/24 h y captopril $25 \mathrm{mg} \mathrm{c} / 24 \mathrm{~h}$, dolor en fosa renal derecha tipo cólico, intermitente, también de un año de evolución, refiere infecciones urinarias recurrentes en una temporalidad de 4 episodios en el último año. A la exploración física no se encuentran datos de importancia. A través del estudio de imagen se evidencian en la urotomografía datos sugestivos de hidronefrosis derecha y se observa un vaso aberrante a nivel de la unión ureteropiélica derecha; así también, se logra observar malrotación renal de forma bilateral, con el eje principal de las unidades renales orientado hacia lateral. (Figura 1)

Se decide realizar gamagrama renal con ácido dietilaminopentacético más furosemida el cual reportó un tiempo medio de eliminación de 18

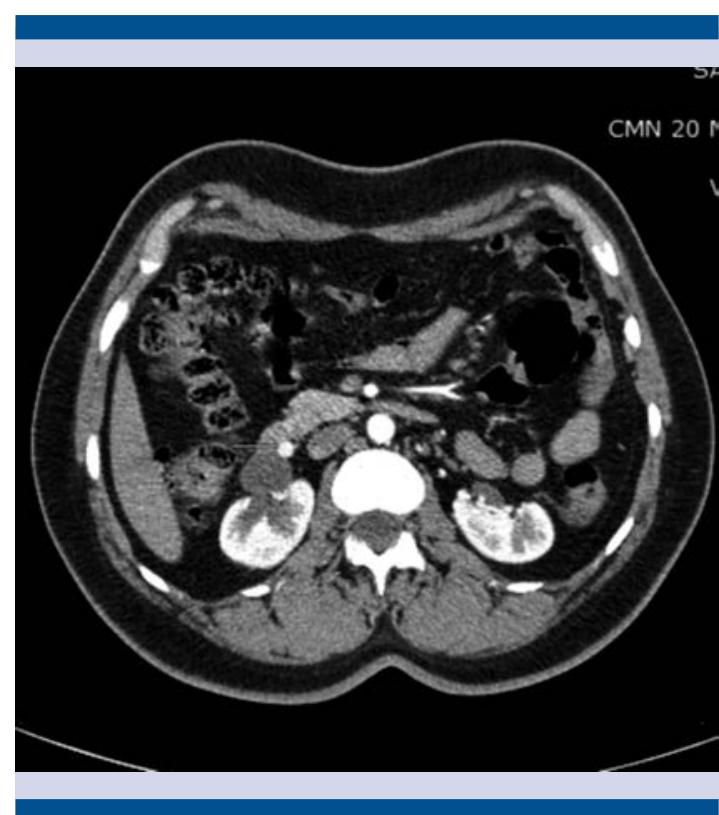

Figura 1. Urotomografía: hidronefrosis, malrotación renal y vaso aberrante por delante de la unión ureteropiélica. minutos para el riñón derecho y 10 minutos para el riñón izquierdo.

Se decidió realizar una plastia ureteropiélica desmembrada con el sistema quirúrgico Da Vinci $^{\circledR}$. Se realizó un abordaje transperitoneal colocando 4 puertos, 2 de $8 \mathrm{~mm}$ para los brazos robóticos y 2 de $12 \mathrm{~mm}$, uno para la cámara y otro para el puerto del ayudante. Se evidenció un vaso aberrante cruzando la cara anterior del uréter (Figura 2), se decidió realizar una plastia desmembrada con resección del segmento ureteral y espatulación del uréter, se colocó catéter JJ a través del puerto del ayudante (Figura 3), la anastomosis al segmento piélico se efectuó con sutura V-loc ${ }^{\circledR}$ 3-0 y se colocó drenaje Drenovac ${ }^{\circledR}$ (Figuras 4 y 5). El tiempo quirúrgico fue de 110 minutos con un sangrado de 50cc, no se reportaron complicaciones en el posoperatorio. La paciente egresó al segundo día de la cirugía. El catéter JJ se retiró tras 6 semanas y se realizó nuevo gamagrama un mes posterior al retiro del catéter. En la consulta de seguimiento a los 3 meses del posoperatorio se realizó el retiro de los medicamentos anti-hipertensivos con adecuado control de la tensión arterial y se obtuvo un adecuado resultado estético.

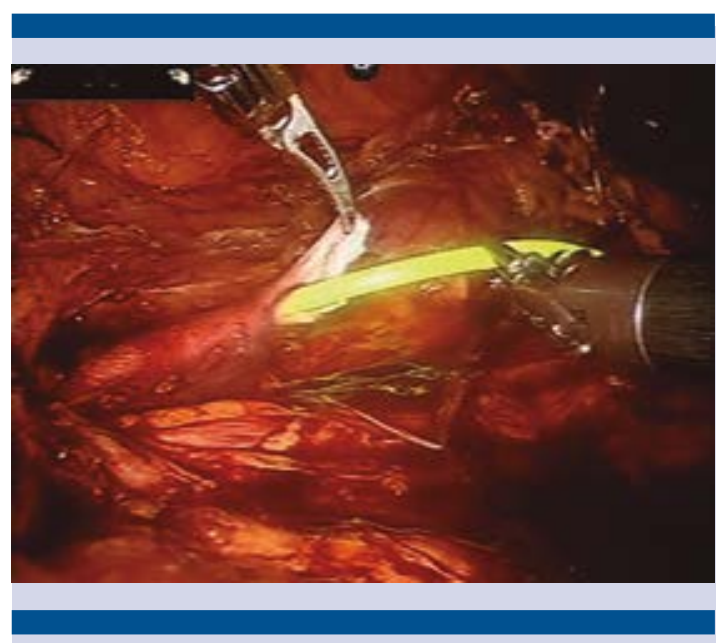

Figura 2. Vaso aberrante por delante de la unión ureteropiélica. 


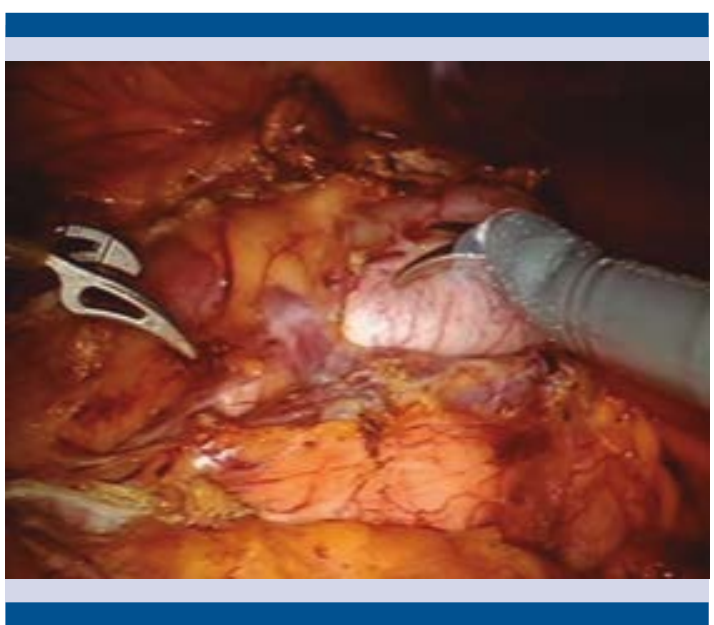

Figura 3. Colocación de catéter a través de un puerto laparoscópico.

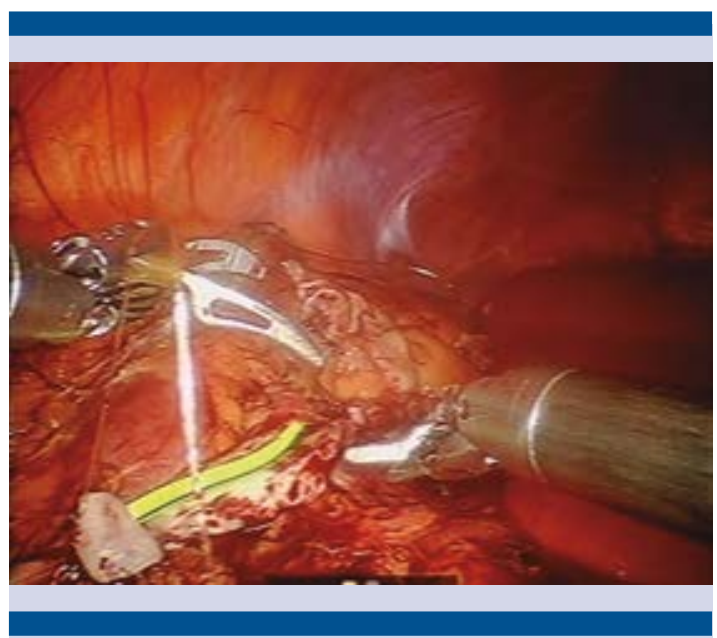

Figura 4. Plastia ureteropiélica con sutura V-Loc.

\section{DISCUSIÓN}

En 1993 Schuessler describió la técnica de plastia desmembrada de la ureteropiélica por laparoscopia donde operando a cinco pacientes sintomáticos logró realizar el procedimiento de forma exitosa, reportando en aquel entonces un tiempo quirúrgico de 3 a 7 horas, donde

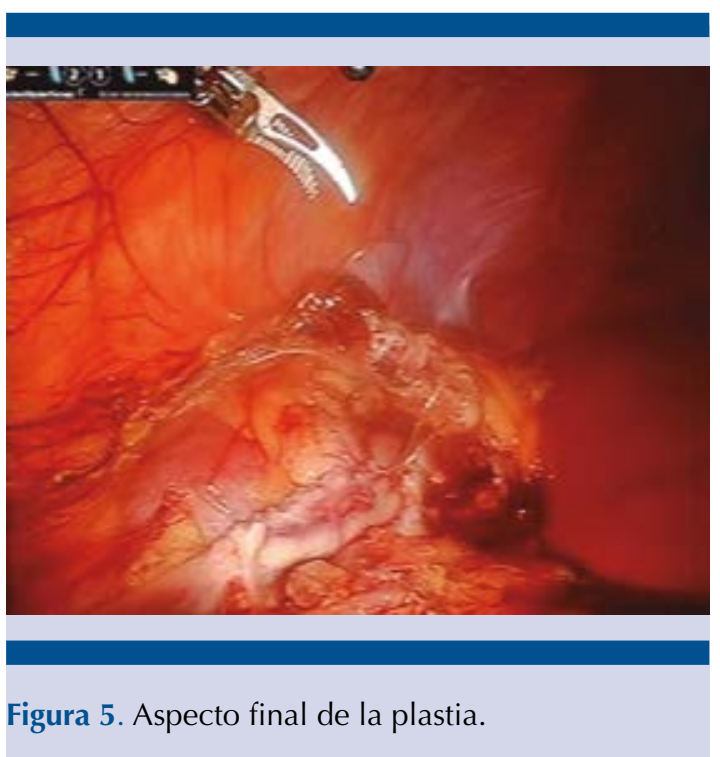

el mayor uso del tiempo se destinó a la sutura laparoscópica (1 a 3 horas). ${ }^{3}$

Con el advenimiento de la cirugía robótica, Sung describió la técnica de plastia de la ureteropiélica laparoscópica asistida con el sistema robótico Zeus en 1999, sin embargo, dicha técnica fue realizada en cerdos y con un abordaje que podríamos llamar combinado, ya que el abordaje del uréter, la disección de la grasa periureteral y de la pelvis renal fue realizado por vía laparoscópica convencional. ${ }^{4}$

En el 2000, Gettman publicó una serie de nueve casos sometidos a plastia desmembrada de la ureteropiélica tipo Anderson-Hynes con ayuda del sistema quirúrgico da Vinci®. En esta serie la media de tiempo operatorio fue de 138 minutos, con un sangrado de $50 \mathrm{ml}$ y reportó buenos resultados con una media de un seguimiento de 4.1 meses. $^{5}$

La plastia desmembrada ha sido el tratamiento de elección para las estenosis de la ureteropiélica desde su descripción en 1949 y desde entonces distintas series han reportado baja tasa de 
complicaciones y una alta tasa de éxito tras el tratamiento, incluso de hasta el $98 \%{ }^{6,7}$

En Estados Unidos, con el paso del tiempo la tendencia a llevar a cabo procedimientos endoscópicos y abiertos para tratar esta patología ha ido decayendo y ha sido remplazada por los procedimientos mínimamente invasivos, pasando de $15.3 \%$ de plastias realizadas por laparoscopia o robot a inicios de la década del año 2000 hasta $55.3 \%$ en $2009 .^{8}$

Debido a los cambios en el tratamiento de esta patología, la tendencia en la actualidad es comparar la efectividad de los procedimientos mínimamente invasivos ( cirugía laparoscópica convencional vs robótica) y esto ha dado lugar a la producción de distintos trabajos que han sido sometidos a revisiones sistemáticas y metanálisis.

En 2009 Braga y colaboradores hicieron un metanálisis que incluyó ocho estudios; se observó disminución significativa de 0.5 días con la cirugía robótica $(\mathrm{p}=0.01)$ y $\sin$ diferencias significativas en el tiempo operatorio $(p=0.15)$, tasa de complicaciones $(p=0.4)$ y tasa de éxito posoperatorio $(p=0.62)$. Sin embargo, entre las limitaciones de su estudio se encontró que no había ensayos con asignación al azar, controlados, y que el acceso a las características demográficas y variables de los estudios revisados fue limitado. Se concluyó que no era posible emitir recomendaciones acerca de la preferencia de un procedimiento en lugar de otro. ${ }^{9}$

Más tarde, en 2014, Autorino y colaboradores Ilevaron a cabo un metanálisis similar pero con la comparación de abordajes mínimamente invasivos versus abiertos; incluyeron 187 artículos y no encontraron diferencias significativas entre la tasa de éxito (RM: 0.96; IC95\%: 0.42-2.21; p $=0.93$ ), la tasa de complicaciones (RM: 0.84; IC95\%: 0.22-3.22; $\mathrm{p}=0.80$ ), necesidad de analgésicos en el posoperatorio $(p=0.19)$ y dismi- nución de 2.86 días a favor de los procedimientos mínimamente invasivos (IC95\%: 3.91-1.45; p $<0.001$ ), así como menor tiempo quirúrgico que favorece a la cirugía abierta (-71.48 min; IC95\%: 48.9-93.9; $\mathrm{p}<0.001)$. En un análisis del subgrupo de cirugía mínimamente invasiva, la única diferencia significativa encontrada entre la cirugía laparoscópica convencional versus la robótica fue la reducción del tiempo quirúrgico que favorece a la cirugía robótica (-27.9 min; IC95\%: 52.5-3.3; $\mathrm{p}=0.03) .{ }^{10}$

En nuestro país no existen series de pacientes con estos procedimientos porque los de la tecnología robótica son de reciente inicio.

La limitación principal en nuestro medio es la dificultad para la adquisición de equipo e insumos para este tipo de procedimientos, junto con un tiempo aún no definido para la curva de aprendizaje para efectuar este tipo de cirugías.

\section{CONCLUSIONES}

En la época de la cirugía mínimamente invasiva la opción debe ser un procedimiento seguro, efectivo y con pocas complicaciones. Con el advenimiento de la cirugía robótica se espera que las dificultades técnicas relacionadas con la visibilidad, la sutura intracorporal y la curva de aprendizaje puedan paliarse con este tipo de tecnología, siempre y cuando esté al alcance del cirujano. La pieloplastia asistida por robot es un método mínimamente invasivo, seguro y efectivo para corregir la estenosis ureteropiélica. Para poder establecer resultados en nuestra población es necesario reunir más pacientes tratados con esta modalidad quirúrgica.

\section{REFERENCIAS}

1. Nakada SY, Hsu TH, Management of Upper Urinary Tract Obstruction. Wein A, Kavoussi LR, Novick AC, Partin AW, Peters CA. Campbell-Walsh Urology. $10^{\text {th }}$ ed. Philadelphia. Elsevier Saunders. 2012. Pp: 1123. 
2. Tasian GE, Casale P. The Robotic-Assisted Laparoscopic Pyeloplasty, Gateway to Advanced Reconstruction. Urol Clin N Am 2015; 42: 89-97.

3. Schuessler WW, Grune MT, Tecuanhuey LV, Preminger GM. Laparoscopic dismembered pyeloplasty. J Urol. 1993; 150(6):1795-9

4. Sung GT, Gill IS, Hsu TH, Robotic-assisted laparoscopic pyeloplasty: a pilot study. Urology 1999; 53(6): 1099-1103

5. Gettman MT, Neururer R, Bartsch G, Peschel R. AndersonHynes dismembered pyeloplasty performed using the da Vinci robotic system. Urology 2002; 60, (3); $509-513$.

6. Anderson JC, Hynes W. Retrocaval ureter: a case diagnosed pre-operatively and treated successfully by a plastic operation. Br J Urol 1949; 21: 209-14.

7. Sivaraman A, Leveillee RJ, Patel MB, et al. Robot-assisted Laparoscopic Dismembered Pyeloplasty for Ureteropelvic
Junction Obstruction: A Multi-institutional Experience. Urology 2012; 79(2): 351- 355.

8. Sukumar S, Sun M, Karakiewicz PI, et al. National trends and disparities in the use of minimally invasive adult pyeloplasty. J Urol 2012; 188: 913-8.

9. Braga LH, Pace K, DeMaría J, et al. Systematic Review and Meta-Analysis of Robotic-Assisted versus Conventional Laparoscopic Pyeloplasty for Patients with Ureteropelvic Junction Obstruction: Effect on Operative Time, Length of Hospital Stay, Postoperative Complications, and .Success Rate. European Urology 2009; 56: 848-858.

10. Autorino R. Eden C. El-Ghoneimi, et al. Robot-assisted and Laparoscopic Repair of Ureteropelvic Junction Obstruction: A Systematic Review and Meta-analysis. European Urology 2014; 65: 430-452.

\section{AVISO PARA LOS AUTORES}

Revista Mexicana de Urología tiene una nueva plataforma de gestión para envío de artículos. En: https://www.revisionporpares.com/index.php/RMUrol podrá inscribirse en nuestra base de datos administrada por el sistema Open Journal System (OJS) que ofrece las siguientes ventajas para los autores:

- Subir sus artículos directamente al sistema.

- Conocer, en cualquier momento, el estado de los artículos enviados, es decir, si ya fueron asignados a un revisor, aceptados con o sin cambios, o rechazados.

- Participar en el proceso editorial corrigiendo y modificando sus artículos hasta su aceptación final. 